\title{
Estrategias didácticas a través de la realidad mixta para el aprendizaje teórico-práctico en estudiantes de educación media superior
}

\author{
Didactic Strategies Through Mixed Reality, for Theoretical-Practical \\ Learning in High School Students
}

\section{Estratégias didáticas por meio de realidade mista para aprendizagem teórico-prática em alunos do ensino médio}

Laura Encarnación De Jesús Universidad de Guadalajara, Centro Universitario de los Valles, México

lauencarnacion@gmail.com https://orcid.org/0000-0003-4902-3361

Suhey Ayala Ramírez

Universidad de Guadalajara, Centro Universitario de los Valles, México suhey.ayala@academicos.udg.mx https://orcid.org/0000-0003-1079-9605

\section{Resumen}

Por las características que presenta, la realidad mixta es una tecnología prometedora dentro del sector educativo. El objetivo de esta investigación fue evaluar la influencia que tiene esta tecnología en el aprendizaje teórico-práctico de estudiantes de educación media superior. El enfoque fue de carácter mixto. Se apoyó de un pretest y postest para evaluar aprendizajes específicos de arquitectura de hardware. Asimismo, se apoyó de las aplicaciones Creator AVR y PC Building. Para evaluar la influencia de la realidad mixta, se utilizó el modelo de aceptación de tecnologías que evalúa los rubros de facilidad, usabilidad y utilidad. Como resultado general, se obtuvo $79.2 \%$ de conocimientos teóricos-prácticos. En el indicador de utilidad, se obtuvo $87 \%$, en el de usabilidad $69 \%$ y en el de facilidad de uso $65 \%$. El estudio permitió observar que el uso de la realidad mixta puede apoyar el aprendizaje teórico-práctico de los estudiantes. 


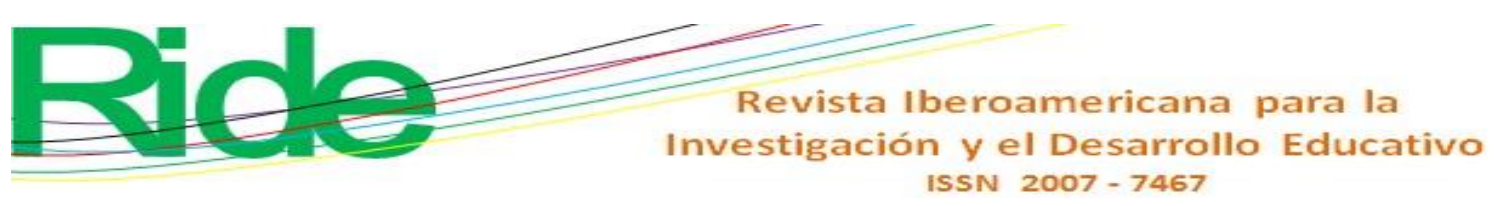

Palabras clave: aprendizaje práctico, realidad aumentada, realidad virtual, tecnologías disruptivas.

\begin{abstract}
Due to its characteristics, mixed reality (MR) is a promising technology within the education sector. The objective of this research was to evaluate the influence that MR has on the theoretical-practical learning of high school students. The study approach was mixed. It was supported by a pre-test and post-test to evaluate specific learning of hardware architecture. Likewise, it relied on the Creator AVR and PC Building applications. To evaluate the influence of MR, the technology acceptance model (TAM) was used, which assesses ease, usability, and utility. As a general result, $79.2 \%$ of theoretical-practical knowledge was obtained. In utility $87 \%, 69 \%$ of usability, and $65 \%$ in ease of use were obtained. The study allowed us to observe that the use of MR can support students' theoretical-practical learning.
\end{abstract}

Keywords: practical learning, augmented reality, virtual reality, disruptive technologies.

\title{
Resumo
}

Pelas suas características, a realidade mista é uma tecnologia promissora no setor educacional. O objetivo desta pesquisa foi avaliar a influência que essa tecnologia exerce na aprendizagem teórico-prática de alunos do ensino médio. A abordagem foi mista. Ele contou com um pré-teste e um pós-teste para avaliar o aprendizado da arquitetura de hardware específica. Também contou com os aplicativos Creator AVR e PC Building. Para avaliar a influência da realidade mista, foi utilizado o modelo de aceitação de tecnologia, que avalia as áreas de facilidade, usabilidade e utilidade. Como resultado geral, obteve-se 79,2\% dos conhecimentos teórico-práticos. No indicador de utilidade, foi obtido $87 \%$, no indicador de usabilidade $69 \%$ e na facilidade de uso $65 \%$. O estudo possibilitou observar que o uso de realidade mista pode subsidiar a aprendizagem teóricoprática dos alunos.

Palavras-chave: aprendizagem prática, realidade aumentada, realidade virtual, tecnologias disruptivas.

Fecha Recepción: Octubre 2020

Fecha Aceptación: Abril 2021 


\section{Introducción}

La evolución que se ha presentado en las tecnologías de información y comunicación (TIC) supone nuevas necesidades y procesos en diferentes áreas. La educación destaca entre estas. Las TIC en los últimos años se han convertido en un componente relevante dentro de los diferentes niveles educativos, y en especial en la educación media superior. Estos avances han permitido experimentar con nuevos dispositivos, recursos y aplicaciones que han abierto un amplio abanico de oportunidades en este sector. Dentro de los avances tecnológicos han surgido herramientas emergentes, innovaciones científicas y avances que han sido y están siendo utilizados en el contexto de la formación pedagógica al servicio de diversos propósitos relacionados con la enseñanza-aprendizaje, además de mostrarse como herramientas disruptivas, puesto que pueden generar cambios en las actividades académicas. En este contexto, surge la realidad virtual (RV), una herramienta que permite sumergir al usuario a otro espacio e interactuar con elementos virtuales a través de un visor.

Centrándose en el contexto educativo, el informe Horizon señala que la RV y las aplicaciones de inmersión han añadido funcionalidades y ofrecen actualmente un mayor potencial para el aprendizaje (Ministerio de Educación, Cultura y Deporte-Instituto Nacional de Tecnologías Educativas y de Formación del Profesorado [Intef], 2017). Otra de las herramientas que recientemente irrumpió en el sector educativo es la realidad aumentada (RA). La RA se caracteriza por superponer una imagen real obtenida a través de una pantalla con imágenes $3 \mathrm{D} \mathrm{u}$ otro tipo de información generada por una computadora (Prendes, 2015, p. 187). Y es a partir de la combinación de estas herramientas, la RV y la RA, que surge la realidad mixta (RM). La RM refiere a la superposición de objetos virtuales en un entorno real que permite a los usuarios interactuar en el mundo real y, al mismo tiempo, con las imágenes virtuales (Vásquez, 2017). Estas herramientas pueden abonar al desarrollo de conocimientos en los estudiantes al cubrir el espectro de la educación tradicional y, a la vez, favorecer la educación basada en competencias. El aprendizaje basado en competencias, además de contribuir a una formación sólida, impulsa las destrezas que son necesarias para que los estudiantes logren ser competitivos desde un punto de vista personal y profesional (Villa y Villa, 2007).

El Sector de Educación de la Oficina de la Unesco en Montevideo (2009) ha externado que la educación debe mejorar y que se deben desarrollar y aplicar modelos educativos que cubran las necesidades de la época actual, así como impulsar el desarrollo de competencias personales, sociales y de aprendizaje que son elementales para que los 


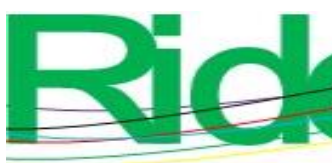

Revista Iberoamericana para la Investigación y el Desarrollo Educativo ISSN 2007 - 7467

estudiantes puedan sobrepasar cualquier desafío que se les pueda presentar en este siglo XXI. Asimismo, los esfuerzos deben encaminarse a que los estudiantes obtengan un aprendizaje integral y conocimientos sólidos que les permitan incursionar en futuros grados académicos, o bien explorar la solución de problemas sociales, ambientales y económicos, entre otros.

Sin embargo, en algunas instituciones de América Latina existen factores externos que impiden que los estudiantes puedan obtener un aprendizaje integral. Por ejemplo, mientras que algo más de $80 \%$ de los habitantes de países desarrollados usan Internet, en países en desarrollo solo se alcanza la cifra de $41 \%$ de conectividad; se trata de déficit en infraestructura de telecomunicaciones, falta de capital humano, pobreza y un entorno inadecuado (Galperín, 2017). En este sentido, las prácticas o experiencias en ocasiones resultan complejas. El sector educativo de México enfrenta una serie de retos dentro del currículo, la evaluación educativa, la didáctica y su aplicación en la vida diaria. Precisamente dentro de este último rubro se localizan las prácticas, componente indispensable para una formación integral, las cuales van de los contenidos a la práctica del estudiante. Estas actividades recaen principalmente en ejercicios prácticos, experiencias e investigaciones ya sea documentales o experimentales (Pérez y Chamizo, 2016).

El propósito de esta investigación fue evaluar de qué manera las estrategias didácticas mediante el uso de RM favorecen el aprendizaje teórico-práctico del campo de la arquitectura de hardware en estudiantes de preparatoria. Un aspecto determinante en este trabajo es que la escuela seleccionada carece de equipo de cómputo para efectuar las prácticas que solicita el plan curricular en el área de informática. Se espera que la investigación abone a la mejora de la didáctica, así como exhortar el uso RV y RA dentro del sector educativo. Así pues, en sintonía con lo hasta aquí mencionado, la pregunta que guio esta investigación fue la siguiente: ¿cómo las estrategias didácticas diseñadas a través de la RM favorecen el aprendizaje teórico-práctico de la arquitectura de hardware de los estudiantes de educación media superior cuya rama de capacitación es la informática? 


\section{Metodología}

Para lograr el objetivo propuesto, se eligió el paradigma multimétodo de combinación. De acuerdo con Bericat (1998), se trata de integrar subsidiariamente un método con otro. En este caso, el cualitativo se apoyó del cuantitativo con el objeto de fortalecer la validez de este último y compensar sus propias debilidades mediante la incorporación de los datos procedentes de la aplicación del método cualitativo. En este sentido, lo que se buscó fue perfeccionar, a través de la implementación de lo cualitativo, solo un componente de la investigación.

En lo que respecta al grado de profundidad, la investigación es explicativa puesto que se buscó probar los vínculos causa-efecto entre la variable independiente, "RM”, y la variable dependiente, "Aprendizaje teórico-práctico". Asimismo, por el diseño y manipulación de dichas variables, es una investigación experimental, dado que fue un proceso que consistió en someter un grupo de estudiantes de preparatoria a un estímulo (diseño de estrategias didácticas mediante el uso de RM) y evaluar los efectos y reacciones que se produjeron en la variable dependiente. Para ello se diseñó un pretest y un postest (Arias, 2006).

Para la variable independiente "RM", se establecieron las categorías de análisis "RV" y "RA". Para estas sus indicadores fueron: Facilidad, Usabilidad y Utilidad. En tanto que, para la variable dependiente, "Aprendizaje teórico-práctico", se establecieron las categorías de análisis: Conocimientos teóricos y Conocimientos prácticos. ${ }^{1}$

Para realizar la intervención con el grupo de estudiantes se diseñó una serie de estrategias didácticas en un objeto de aprendizaje con base en un modelo de diseño instruccional congruente con la mediación de las TIC y la teoría del aprendizaje constructivista que permitiera observar el cambio en la variable dependiente.

\section{Instrumentos de recolección de datos}

\section{Instrumentos cuantitativos}

Para el análisis de las categorías Conocimientos teóricos y Conocimientos prácticos se utilizó un pretest y un postest sobre arquitectura de hardware (véase tabla 4) que permitió evidenciar los conocimientos previos de los estudiantes y los resultados después de aplicar las estrategias didácticas mediante el uso de la RM.

\footnotetext{
${ }^{1}$ Se hizo esta división entre el conocimiento teórico y práctico para facilitar el análisis de los datos observados en la investigación, sin embargo, se reconoce que hay un vínculo estrecho entre estos dos conceptos. De hecho, para algunos autores son indivisibles.
} 


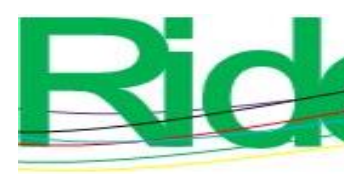

Revista Iberoamericana para la Investigación y el Desarrollo Educativo ISSN 2007 - 7467

Asimismo, se utilizó el test (véase tabla 5) del Technology Acceptance Model (TAM) de Davis (1986, citado en Davis, Bagozzi, y Warsaw, 1989), que midió las variables "RV" y "RA" a través de sus indicadores: Facilidad, Usabilidad y Utilidad. Este modelo ha sido utilizado para evaluar la aceptabilidad de tecnologías por los usuarios. Con la finalidad de mejorar la fiabilidad de los resultados, se apoyó del estadístico de alfa Cronbach. El modelo de TAM se compuso de nueve ítems que ofrecían cinco opciones según la siguiente escala de valores: Totalmente en desacuerdo $=1$, Desacuerdo $=2$, Indiferente $=3$, De acuerdo $=4$ y Totalmente de acuerdo $=5$.

\section{Instrumentos cualitativos}

La integración del método cualitativo se realizó para incorporar datos complementarios sobre la interacción de los estudiantes con la tecnología. Esto se hizo a través de la observación del participante. Para el vaciado de los datos y su posterior análisis, se apoyó de una lista de cotejo (véase tabla 6), que consistió en llevar el control de las prácticas que realizaron los estudiantes, de acuerdo con los temas planteados, con el apoyo de las aplicaciones de Creator AVR (gama de RA) y PC Building (gama de RV). Se procuró recabar todos los datos posibles a través de la observación directa y plasmarlos en el apartado de observaciones de la lista de cotejo, esto para generar una mejor interpretación en las categorías Conocimientos teóricos y Conocimientos prácticos.

\section{Participantes}

La muestra fue extraída de estudiantes de educación media superior a través de un muestreo no probabilístico por conveniencia e intencional. Asimismo, se consideró a estudiantes que habían cursado la materia de Arquitectura de Hardware, esto es, alumnos de la capacitación de informática de $5 .{ }^{\circ}$ semestre, quienes se encontraban en un rango de edad de 16 a 17 años. El total de la muestra fue de 13 estudiantes, que constó de cinco hombres y ocho mujeres.

\section{Método de análisis de los datos}

Para el análisis de los datos cuantitativos (pretest, postest y modelo de TAM), se apoyó de formularios creados en Google Forms, lo cual permitió su posterior análisis con apoyo del paquete estadístico SPSS. Así, se generaron gráficos descriptivos según los criterios de análisis. En cuanto al modelo de TAM, cabe mencionar que se apoyó del alfa Cronbach para comprobar la fiabilidad de los instrumentos aplicados y, por ende, de los resultados obtenidos. En el caso del instrumento cualitativo, se utilizó la lista de cotejo 
para hacer el vaciado de los datos derivados de la observación y su posterior análisis de acuerdo con los criterios establecidos en dicho instrumento.

\section{Descripción del proceso de implementación}

La implementación de estrategias didácticas con apoyo de la RM se basó en el modelo de cinco fases: análisis, diseño, desarrollo, implementación y evaluación (Addie). Este proceso se interrelaciona de manera transversal con aportes teóricos del constructivismo. El Addie busca que el estudiante sea el protagonista de su aprendizaje (Jonassen, Peck y Wilson, 1999). El diseño instruccional que se utilizó considera el aprendizaje en ambientes virtuales y de forma directa, lo que hace que este proyecto se haya mostrado en una formación directa y de ambientes virtuales, tal como se plasma en la figura 1 .

Figura 1. Diseño instruccional basado en una formación directa según Hirumi

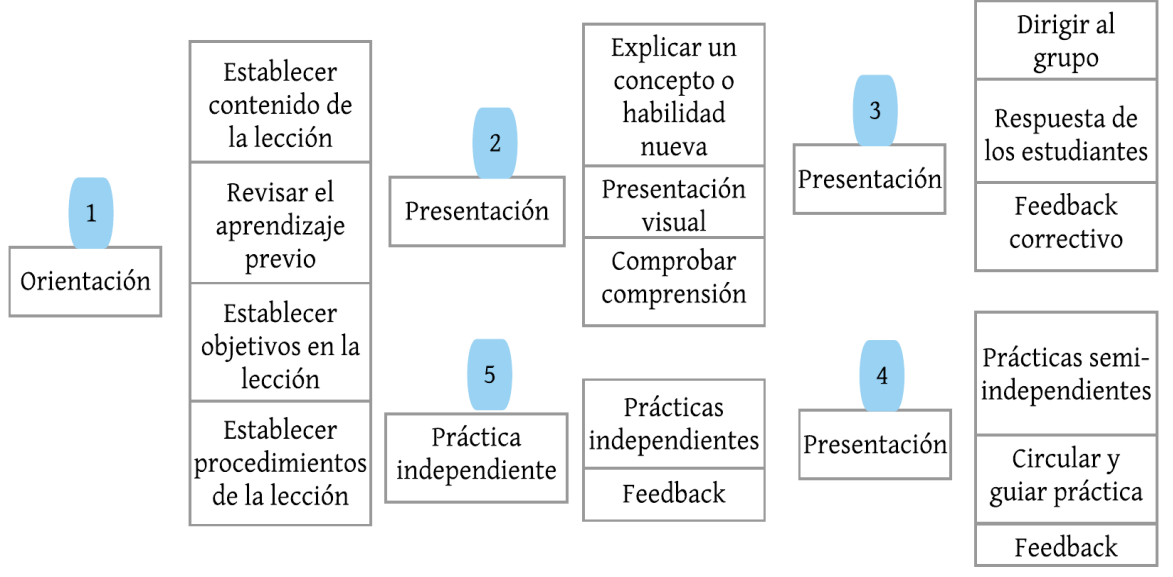

Fuente: Villafañe (2009)

Broderick (2001) define al diseño instruccional como la ciencia para crear ambientes de trabajo; allí se detallan instrucciones, se proporcionan materiales claros y efectivos que favorezcan al desarrollo de habilidades y destrezas por parte de los alumnos. De acuerdo con lo antes mencionado, la implementación de este proyecto se apoyó de un diseño instruccional que se adaptó a un objeto de aprendizaje que constó de materiales e instrucciones, además de apoyarse de la RM. A continuación, se detalla sobre cada una de las fases del Addie. 


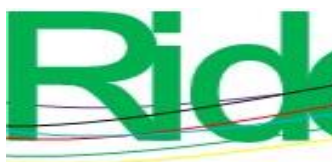

Revista Iberoamericana para la Investigación y el Desarrollo Educativo ISSN 2007 - 7467

laboratorio para estos fines. El proceso de implementación se efectuó como se muestra en la tabla 1, considerando y aprovechando que la mayoría de los estudiantes contaba con un dispositivo móvil.

Tabla 1. Proceso de implementación

\begin{tabular}{|c|c|}
\hline \multicolumn{2}{|r|}{ Presentación "Estrategias didácticas de Arquitectura de Hardware" } \\
\hline Sesión 1 & $\begin{array}{l}\text { Se explicó a grandes rasgos la finalidad de la investigación y la } \\
\text { participación que tendrían los discentes. Además, se dio una } \\
\text { demostración de RV y RA con el fin de aproximarlos a una experiencia } \\
\text { con estas herramientas. Asimismo, se solicitó a los estudiantes contestar } \\
\text { el pretest sobre arquitectura de hardware y firmar la carta de } \\
\text { consentimiento para que conocieran los pormenores de su participación } \\
\text { dentro de la intervención. }\end{array}$ \\
\hline Sesión 2 & $\begin{array}{l}\text { En esta sesión, los estudiantes tuvieron un segundo acercamiento con las } \\
\text { RV y RA, con el fin de adaptarlos al uso de estas tecnologías. Además, } \\
\text { se les proporcionó los recursos necesarios para que pudieran crear sus } \\
\text { gafas Google Cardboard en el momento que lo desearan después de la } \\
\text { intervención y no se limitaran por las gafas costosas que existen en el } \\
\text { mercado. }\end{array}$ \\
\hline Sesión 3 & $\begin{array}{l}\text { Componentes básicos de una computadora } \\
\text { En este apartado se apoyó de la aplicación de Creator AVR y de los } \\
\text { dispositivos móviles. Los estudiantes interactuaron con la RA e } \\
\text { identificaron las partes básicas de una computadora. }\end{array}$ \\
\hline Sesión 4 & $\begin{array}{l}\text { Partes internas y externas de computadora } \\
\text { En este apartado los estudiantes contaron con recursos disponibles en el } \\
\text { Objeto de Aprendizaje, como Slideshare y Educaplay para interactuar } \\
\text { con ejercicios prácticos, además de estar acompañados de la aplicación } \\
\text { de Creator AVR, para la familiarización directa con las partes internas y } \\
\text { externas que compone una computadora. }\end{array}$ \\
\hline Sesión 5 & $\begin{array}{l}\text { En este apartado los estudiantes conocieron la secuencia correcta de } \\
\text { ensamblar una computadora a través del software de PC Building }\end{array}$ \\
\hline
\end{tabular}




\begin{tabular}{|l|l|}
\hline Simulator. Esta herramienta educativa tiene la comodidad de simular un \\
laboratorio virtual, donde los discentes tuvieron la facilidad de ingresar \\
para ensamblar una computadora. Asimismo, se pudo llevar la secuencia \\
de ensamble con la RA a través del software Creator AVR. Al concluir la \\
implementación de RM, se aplicó una evaluación general con la finalidad \\
de medir el aprendizaje que se pudo generar a través de estas \\
herramientas.
\end{tabular}

Fuente: Elaboración propia

\section{Evaluación}

Cabe resaltar que en cada unidad se presentaron actividades lúdicas (involucramiento de RA, crucigramas, sopas de letras, adivina qué es y actividades de Educaplay) $^{2}$ para reforzar el aprendizaje que se mostraron en el proceso. Asimismo, al concluir la implementación de RM, se aplicó el postest sobre arquitectura de hardware para evaluar las categorías Conocimientos teóricos y Conocimientos prácticos y el test del modelo de TAM para evaluar los indicadores Utilidad, Usabilidad y Facilidad de uso. Todo esto con el fin último de medir el aprendizaje generado a través de la RM, así como el porcentaje de aceptabilidad hacia estas herramientas.

\section{Resultados}

La población analizada constó de la participación de 13 estudiantes organizados en un solo grupo. En primera instancia, se apoyó del pretest, que se enfocó en medir los conocimientos previos adquiridos en $4^{\circ}$ semestre, así como también se basó en el análisis de conocimientos adquiridos posterior a la implementación. Como ya lo dijimos, esta instancia se enfoca en las categorías Conocimientos teóricos y Conocimientos prácticos. Al concluir la implementación, también se aplicó el test del modelo de TAM, que evaluó la aceptabilidad de tecnologías considerando los indicadores Usabilidad, Utilidad y Facilidad.

\footnotetext{
${ }^{2}$ Educaplay es una plataforma que permite a los usuarios crear actividades educativas multimedia con un resultado atractivo y profesional como mapas, adivinanzas, crucigramas, etcétera.
} 


\section{Resultados del test de arquitectura de hardware}

El proceso de recolección de datos consistió en el análisis de la aplicación del pretest (véase tabla 4), compuesto de 13 ítems, preguntas generales de la materia Arquitectura de Hardware. De acuerdo con los resultados obtenidos, se observó que los estudiantes solo alcanzaron $53 \%$ en conocimientos sólidos en la materia cursada en el semestre anterior. Los valores con mayor grado de pertinencia se registraron en áreas referidas a la información, comunicación y con una disminución en casos prácticos (véase figura 3).

Figura 3. Resultados de conocimientos previos de la materia de Arquitectura de Hardware

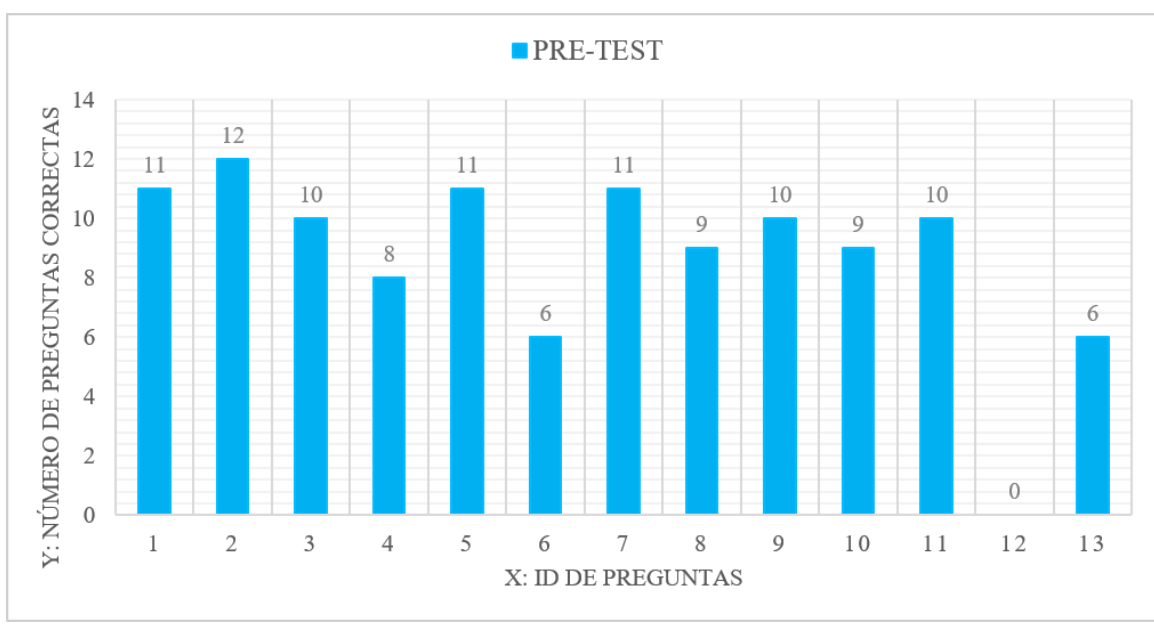

Fuente: Elaboración propia

Como se puede observar, existe disminución en el ítem seis. Esta representa la identificación de las partes de la motherboard (placa madre). De acuerdo con los resultados, solo $46.15 \%$ (seis alumnos) posee conocimientos sólidos en la identificación de esta.

En cuanto al ítem 12, ¿Alguna vez ha ensamblado una computadora?”, ninguno de los participantes respondió afirmativamente, es decir, no ejecutaron ciertas prácticas que se plantean en el currículo.

En el ítem 13, “¿Identifica los pasos y las medidas correctas para ensamblar una computadora?", como se puede observar, $46.15 \%$ (seis alumnos) expresó conocimientos en cuanto al proceso de ensamble, es decir, considera las medidas adecuadas para este, en otras palabras, basados únicamente en los conocimientos teóricos.

Para conocer la influencia que se tuvo tras la implementación, se aplicó el postest de arquitectura de hardware. En la figura 4 se puede observar que los estudiantes obtuvieron un incremento en la información, la comunicación y, lo más importante, en 


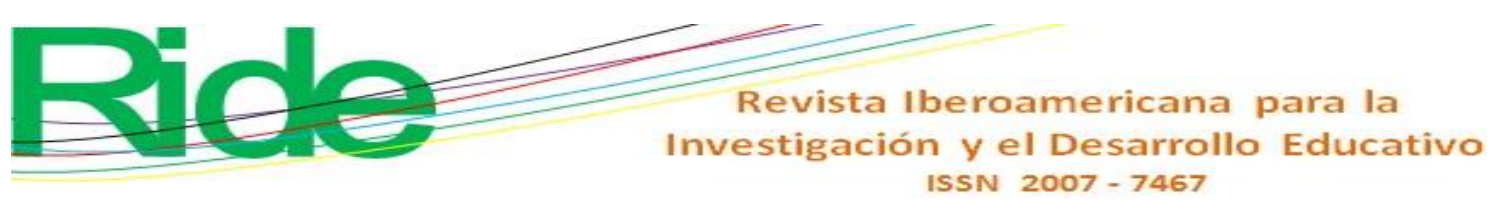

los casos prácticos con $79.2 \%$ de resultado. Es decir, se logró un incremento de $26.03 \%$ con apoyo de la RM para el aprendizaje práctico.

Figura 4. Resultados del pretest y postest

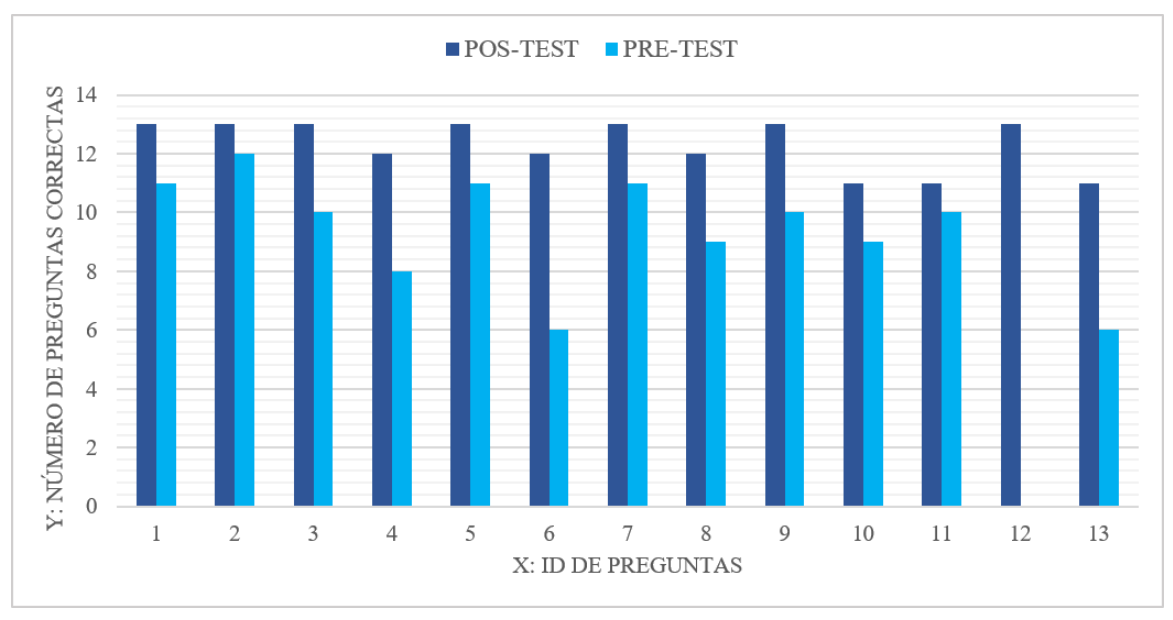

Fuente: Elaboración propia

\section{Resultados de la lista de cotejo}

En cuanto a los resultados de la lista de cotejo, cabe mencionar que se utilizó para llevar un control de las prácticas efectuadas por parte de los estudiantes y apoyadas de las aplicaciones de PC Building Simulator y Creator AVR. El propósito fue llevar el registro y control sobre las recomendaciones y pasos para ensamblar de manera correcta una PC. Este instrumento sirvió para reforzar y corroborar los resultados del postest de arquitectura hardware, principalmente en los ítems 6, 12 y 13. Los datos recogidos son favorables, ya que los estudiantes se vieron en la necesidad de conocer todos los pasos para que la aplicación los dejara avanzar y así finalizar la actividad. Es posible decir que $90 \%$ de los estudiantes conoció los pasos correctos, así como las medidas a considerar antes de ensamblar una computadora.

\section{Resultados del modelo de TAM (indicadores Facilidad, Usabilidad y Utilidad)}

Para conocer los resultados de los indicadores Facilidad, Usabilidad y Utilidad se aplicó una encuesta (véase tabla 5), cuyo sustento fue el modelo de TAM, que se encarga del estudio de la aceptación de tecnología (variables "RA" y "RV"). La encuesta se compuso de nueve ítems en el que se consideró la escala de valores: Totalmente en desacuerdo $=1$, Desacuerdo $=2$, Indiferente $=3$, De acuerdo $=4$ y Totalmente de acuerdo 
$=5$. Asimismo, para tener confiabilidad en los resultados, se interpretó con el alfa de Cronbach, el cual se calculó usando la fórmula 1.

$$
a=\frac{k}{k-1}\left(1-\frac{\sum V_{i}}{V_{t}}\right)
$$

El alfa de Cronbach es un coeficiente que se utiliza para medir la consistencia interna; se basa en el promedio de las correlaciones entre los ítems. En otras palabras, el alfa de Cronbach es el promedio de correlaciones entre los ítems que hacen parte de un instrumento. El mínimo es de 0.70; por debajo de este valor la escala utilizada es baja. Mientras que un resultado por encima de 0.90 apunta a que existe redundancia o duplicación. Regularmente se prefieren valores entre 0.80 y 0.90 (Cronbach, 1951). En la tabla 2 se presentan los resultados de los indicadores Facilidad, Usabilidad y Utilidad de las categorías "RV" y "RA". De acuerdo con los con los datos y la interpretación del alfa de Cronbach, los estudiantes obtuvieron 0.81 de confiabilidad (véase tabla 3), lo que quiere decir que se alcanzó una evaluación de nivel buena.

Tabla 2. Interpretación del modelo de TAM con el alfa de Cronbach

\begin{tabular}{|c|c|c|c|c|c|c|c|c|c|c|}
\hline & Ítem1 & Ítem2 & Ítem3 & Ítem4 & Ítem5 & Ítem6 & Ítem7 & Ítem8 & Ítem9 & Suma \\
\hline E1 & 1 & 4 & 1 & 4 & 2 & 2 & 1 & 2 & 1 & 18 \\
\hline E2 & 1 & 1 & 1 & 1 & 4 & 1 & 1 & 1 & 1 & 12 \\
\hline E3 & 1 & 1 & 1 & 1 & 2 & 2 & 1 & 1 & 1 & 11 \\
\hline E4 & 2 & 4 & 1 & 4 & 5 & 2 & 1 & 1 & 3 & 23 \\
\hline E5 & 1 & 4 & 2 & 4 & 4 & 2 & 2 & 1 & 2 & 22 \\
\hline E6 & 2 & 2 & 2 & 2 & 4 & 2 & 1 & 2 & 2 & 19 \\
\hline E7 & 2 & 2 & 2 & 2 & 3 & 2 & 3 & 2 & 3 & 21 \\
\hline E8 & 1 & 1 & 1 & 1 & 5 & 1 & 1 & 1 & 1 & 13 \\
\hline E9 & 1 & 4 & 1 & 4 & 2 & 1 & 2 & 1 & 2 & 18 \\
\hline E10 & 1 & 1 & 2 & 1 & 1 & 1 & 1 & 1 & 1 & 10 \\
\hline E11 & 3 & 4 & 2 & 4 & 4 & 2 & 3 & 3 & 2 & 27 \\
\hline E12 & 1 & 3 & 2 & 3 & 4 & 2 & 1 & 2 & 2 & 20 \\
\hline E13 & 3 & 3 & 2 & 3 & 4 & 2 & 1 & 3 & 3 & 24 \\
\hline Varianza & 0.5562 & 1.6213 & 0.2485 & 1.6213 & 1.4675 & 0.213 & 0.5562 & 0.5444 & 0.5917 & \\
\hline
\end{tabular}

Fuente: Elaboración propia 
Tabla 3. Interpretación de los resultados del modelo de TAM basada en la ecuación del alfa de Cronbach

\begin{tabular}{|l|r|}
\hline A (alfa) $=$ & 0.810241 \\
\hline K (número de ítems) $=$ & 9 \\
\hline $\begin{array}{l}\text { Vi (varianza de cada } \\
\text { ítem })=\end{array}$ & 7.420118 \\
\hline Vt (varianza total) $=$ & 26.52071 \\
\hline
\end{tabular}

Fuente: Elaboración propia

Siguiendo los resultados analizados de los indicadores de Facilidad, Usabilidad y Utilidad, la respuesta de integración de las categorías "RV" y "RA" se mostró de manera positiva por parte de los estudiantes. Para una mejor interpretación en la figura 5 se muestran los resultados sobre cada uno de los indicadores mencionados. En lo que concierne al indicador Facilidad (totalmente de acuerdo y de acuerdo), hay una percepción positiva con $65 \%$, es decir, la cantidad de estos participantes no encontró dificultad en el uso de la RM. Sin embargo, $35 \%$ expresó dificultad en el proceso de actividades que involucraban la RM debido a que no se encontraban familiarizados con este tipo de recursos.

Figura 5. Interpretación del modelo de TAM

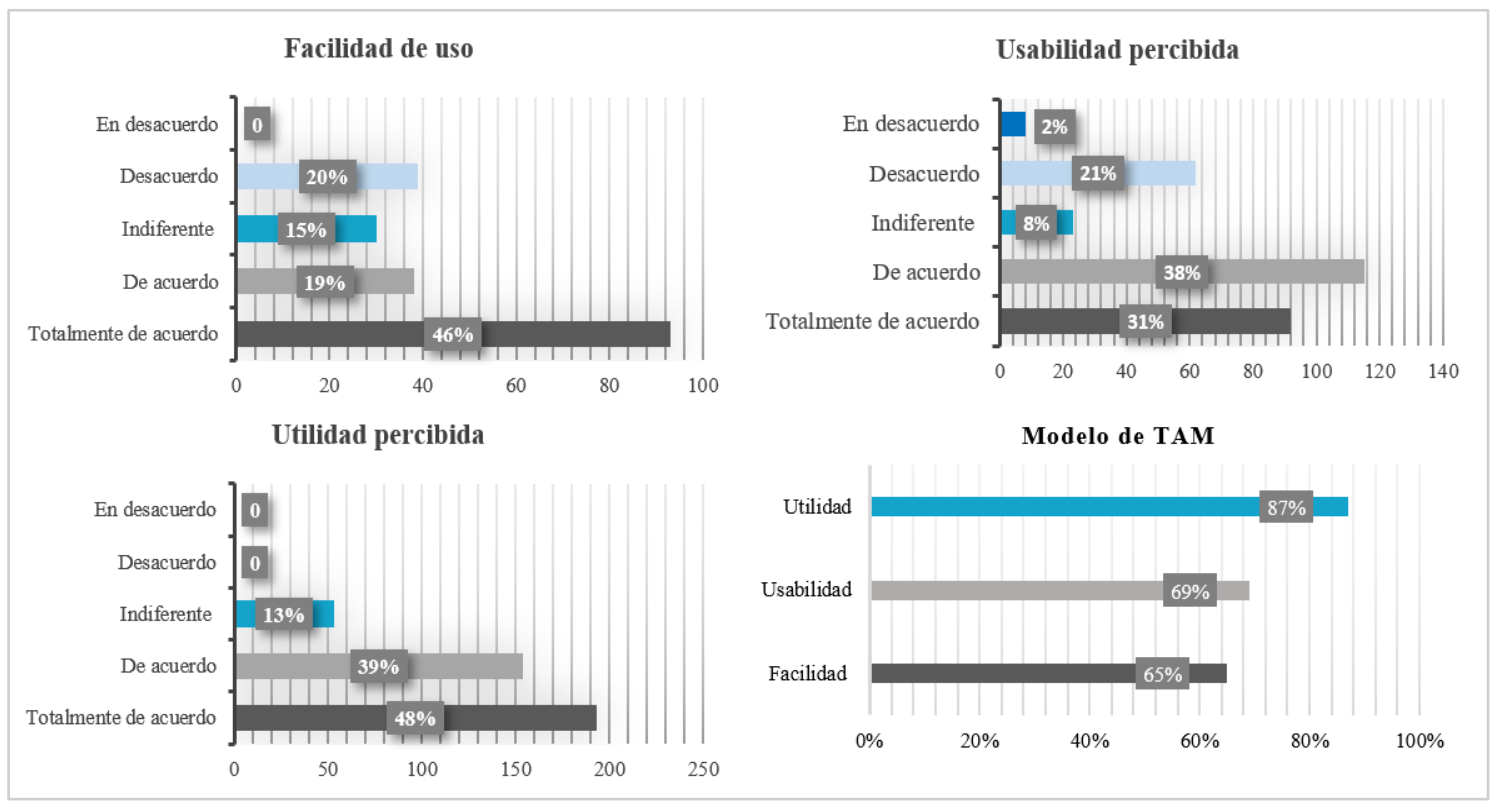

Fuente: Elaboración propia

En cuanto al indicador Usabilidad (véase figura 5), se obtuvo $69 \%$ en la interactividad y comprensión de contenido, sin embargo, $31 \%$ indicó indiferencia hacia el contenido de la RV y RA. Esto se debe al desconocimiento de la RM, a pesar de ello, 


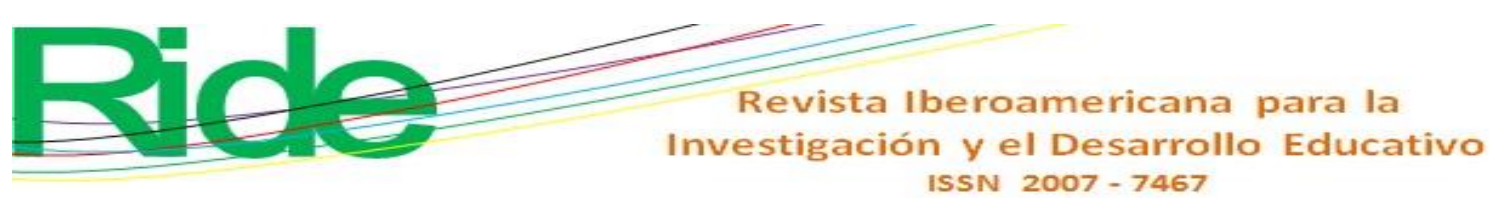

manifestaron que les gustaría que los contenidos apoyados a través de la RM formaran parte de su formación académica en diversas materias.

Por otro lado, el indicador Utilidad (véase figura 5) registró un efecto de $87 \%$ (totalmente de acuerdo y de acuerdo) en la comprensión de los temas tocados de la materia de Arquitectura de Hardware con el apoyo de la RM.

\section{Discusión}

La presente investigación se enfocó en evaluar de qué manera el uso de estrategias didácticas apoyadas de la RM influye en el aprendizaje teórico-práctico de estudiantes de educación media superior, particularmente en quienes tiene como capacitación la informática y, siendo aún más precisos, en el tópico de arquitectura de hardware. Aunado a que se apoyó de un objeto de aprendizaje que fungió como mediador para la disposición de recursos y actividades que sirvieron para fortalecer los conocimientos apoyados de la RM.

Luego de haber procedido a la implementación, y con base en la recopilación de datos, se puede expresar que el uso de estrategias didácticas apoyadas de la RM, mediado por plataformas que contengan recursos y actividades para reafirmar lo aprendido, propicia el aprendizaje significativo de los discentes. Igualmente, motiva e incentiva el proceso de aprendizaje a través de estas herramientas por las características que presenta. En este sentido, se podría decir que investigaciones como esta se convierten en una pieza clave para entender cómo podría hacerse uso de la RM para el aprendizaje teóricopráctico en espacios que carezcan de equipo especializado de laboratorio.

Los resultados obtenidos llevan a pensar que el uso de la RM sí mejora el aprendizaje teórico-práctico, lo cual se evidencia en el ítem 6, 12 y 13 (véase tabla 4). Estos resultados coinciden con los hallazgos de Villarejo (2019) y Hamada, Mohamed, Mohamed y Youssef (2018).

En cuanto a la evaluación de la aceptabilidad de RA y RV, se puede afirmar que, respecto al indicador Facilidad, compuesto de dos ítems (véase tabla 5), permitió evidenciar la comodidad de interactuar con los componentes y el proceso de actividades apoyados de la RA y RV, así como el manejo de dispositivos móviles durante la visualización de imágenes en $3 \mathrm{D}$. Los resultados muestran una recepción positiva con $65 \%$.

Para el indicador Usabilidad se consideraron tres ítems que hicieron referencia a la interactividad, comprensión de contenido y diseño de contenido. Aquí se puede expresar que los estudiantes mostraron comodidad para su aprendizaje a través de estas 


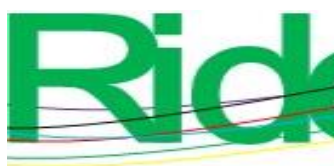

Revista Iberoamericana para la Investigación y el Desarrollo Educativo ISSN $2007-7467$

herramientas. Esto evidentemente se puede corroborar en los resultados obtenidos: 69 \% manifestó aceptabilidad en cuanto a la usabilidad.

Para el indicador Utilidad se consideraron cuatro ítems (véase tabla 5), los cuales evaluaron si la RM apoyó la formación académica de los estudiantes, además de despertar la motivación de aprendizaje por las características que presentan estas herramientas. De acuerdo con los resultados obtenidos, se puede expresar que fueron aceptadas de manera favorable; aquí se registró $87 \%$ de utilidad. Estos resultados coinciden con la investigación de Mojerón (2018) y Lasheras (2018).

Finalmente, se puede expresar el presente documento aporta datos relevantes que podrían ser utilizados para considerar estrategias didácticas apoyadas de la RM, con el fin de propiciar un aprendizaje teórico práctico que les permita desarrollar habilidades y destrezas que requieren para la inserción laboral o futuros grados académicos, así como con el de motivar e incentivar a los estudiantes a la adquisición de una formación integral.

\section{Conclusiones}

El objetivo de este estudio fue, además de evaluar la efectividad de las estrategias didácticas mediante el uso de RM, corroborar la aceptabilidad del uso de herramientas tecnológicas (en este caso la RM) como recursos didácticos para el aprendizaje. Para ello se consideró el modelo de TAM, el cual se encargó de evaluar las variables "RA" y "RV" a través de los indicadores ya mencionados.

Así pues, es posible responder que el efecto de evaluación fue positivo, ya que se lograron adquirir conocimientos teóricos-prácticos con el apoyo de la RM, muestra de ello son los resultados obtenidos de la prueba de arquitectura de hardware y una lista de cotejo donde se pudo visualizar un aumento significativo de aprendizaje teórico-práctico. Esta mejoría se pudo detectar por el pretest y el postest. Como primer resultado se obtuvo $53 \%$ en conocimientos teóricos, sin considerar los conocimientos prácticos. Posterior a la implementación, se tuvo un incremento de $79.2 \%$ referido a la información, comunicación y, lo más importante, en los casos prácticos: un incremento de $26.03 \%$ con apoyo de la RM para el aprendizaje práctico.

Se concluye que el involucramiento de RM en el sector educativo podría apoyar las prácticas de ensamblaje de una computadora, además de apoyar las condiciones de aprendizaje y la inclusión de tecnologías emergentes. Por otro lado, esta herramienta podría ser replicada en materias que requieran de uso práctico de equipos especializados. En este sentido, el Equipo aCanelma (11 de julio de 2016) expresa que la RV puede ser 


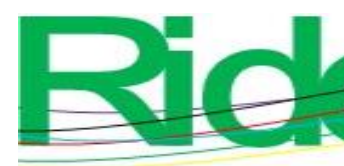

Revista Iberoamericana para la

Investigación y el Desarrollo Educativo

ISSN 2007 - 7467

utilizada en un enfoque educativo basado en experiencias de aprendizaje centradas en sumergir a los discentes en lugares de patrimonio local, cultural, paisajístico, etcétera. Además, puede ser utilizada en diversas materias como: artes del lenguaje, matemáticas, estudios sociales, ciencias, etcétera. Asimismo, se resalta que no necesariamente se requiere de visores costosos para la visualización de los objetos $3 \mathrm{D}$, ya que se puede hacer uso de los visores de Google Cardboard, ${ }^{3}$ visores que permiten experimentar la RV de manera sencilla y divertida.

Por otro lado, es relevante mencionar que la RM permite un aprendizaje significativo, atractivo y motivacional en los estudiantes, prueba de ello se vio reflejado en la encuesta aplicada del modelo de TAM, que evaluó la aceptabilidad de la RM, así como también el objeto de aprendizaje que contenía recursos y actividades que ayudaron a reforzar el aprendizaje de los discentes. Esto se vio reflejado en los indicadores Facilidad, Usabilidad y Utilidad; hubo datos significativos en cada una de estos: 87 \%, $69 \%$ y $65 \%$ respectivamente.

En cuanto a la pregunta trazada para esta investigación, ¿cómo las estrategias didácticas diseñadas a través de la RM favorecen el aprendizaje teórico-práctico sobre arquitectura de hardware en los estudiantes de educación media superior de la capacitación de informática?, siguiendo los resultados obtenidos, es posible responder que la RM produce efectos positivos en el aprendizaje teórico-práctico en los estudiantes, principalmente en el conocimiento práctico, que fue el tema medular de esta investigación.

El análisis de las variables mencionadas pone en evidencia la contribución que tiene la RM para la adquisición de conocimientos teóricos y conocimientos prácticos en las instituciones donde se carece de equipo especializado, se tienen problemas de tiempo o seguridad. La RM influye en la motivación y contribuye en el desarrollo de habilidades y destrezas que requieren los estudiantes en esta era digital, según los resultados alcanzados. En general, la percepción de los discentes a partir del uso de estas herramientas para apoyar su aprendizaje ha sido favorable, así como también ha despertado el interés y la motivación por la forma lúdica en que estas herramientas se presentan.

Para finalizar, hay que mencionar que una de las fortalezas de esta investigación fue la intervención planeada y apoyada de un diseño instruccional. En este caso, se utilizó el modelo Addie. Concretamente, el Addie se adaptó de acuerdo con las necesidades de los estudiantes y docentes.

\footnotetext{
${ }^{3}$ Los visores Google Cardboard son gafas de realidad virtual realizadas con cartón y lentes de distancia focal y son utilizadas con un dispositivo móvil.
} 


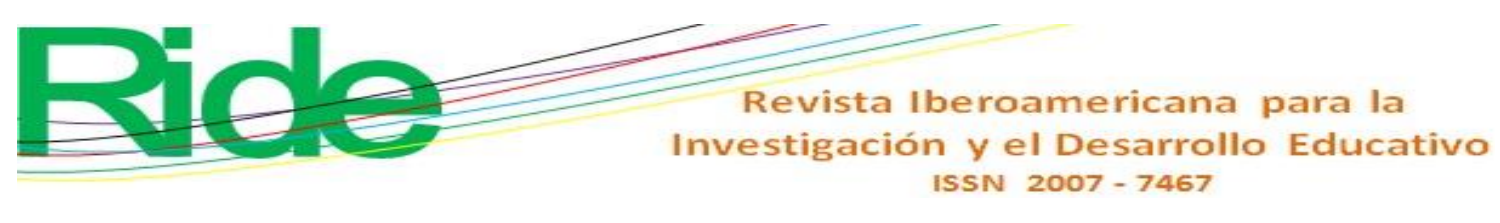

\section{Futuras líneas de investigación}

Para futuras investigaciones se sugiere explorar otras instituciones con las mismas condiciones para comparar resultados. Asimismo, el estudio podría aplicarse con grupos control que permitan un análisis profundo. Otra línea interesante podría ser la incidencia en el uso de estas herramientas por parte de los docentes, es decir, cómo pueden adaptar este tipo de tecnología para mediar el proceso de enseñanza-aprendizaje. Por último, sería interesante el uso de estrategias didácticas apoyadas de la RV o RA en otras disciplinas como, por ejemplo, estimular el pensamiento matemático en los estudiantes.

\section{Referencias}

Arias, F. G. (2006). El proyecto de investigación. Introducción a la investigación cientifica. (5. ${ }^{\mathrm{a}}$ ed.). Venezuela: Editorial Epistema.

Bericat, E. (1998). La integración de los métodos cuantitativo y cualitativo en la investigación social. Significado y medida. Barcelona: Ariel.

Broderick, C. (2001). Instructional systems design: What it's all about. Training Magazine, 25(1), 1-15.

Cronbach, L. (1951). Coefficient alpha and the internal structure of tests. Psychometrika, 16(3), 297-334.

Davis, F., Bagozzi, R. and Warsaw, P. (1989). User Acceptance of Computer Technology: A Comparison of Two Theoretical Models. Management Sciences, 35(8), 9831003.

Equipo aCanelma. (11 de julio de 2016). Preguntas y respuestas en torno a la realidad virtual en educación. aCanelma. Recuperado de https://www.acanelma.es/search/label/recursos.

Galperín, H. (2017). Sociedad digital: brechas y retos para la inclusión digital en América Latina y el Caribe. En Organización de las Naciones Unidas para la Educación, la Ciencia y la Cultura y Oficina Regional de Ciencias de la Unesco para América Latina y el Caribe, Sociedad digital: brechas y retos para la inclusión digital en América Latina y el Caribe. Montevideo, Uruguay: Organización de las Naciones Unidas para la Educación, la Ciencia y la Cultura y Oficina Regional de Ciencias de la Unesco para América Latina y el Caribe. Recuperado de https://unesdoc.unesco.org/ark:/48223/pf0000262860. 
Hamada, E. K., Mohamed, E. A., Mohamed, S. and Youssef, M. (2018). Toward an occluded augmented reality framework in e-learning platforms for practical activities. Journal of Engineering Science and Technology, 13(2), 394-408.

Jonassen, D., Peck, K. and Wilson, B. (1999). Learning with technology: A Constructivist Perspective. Upper Saddle River, United States: Merrill / Prentice Hall.

Lasheras, C. (2018). La realidad aumentada como recurso educativo en la enseñanza de español como lengua extranjera. Propuesta de intervención a partir de un manual. (Tesis de maestría). Universidad Internacional de la Rioja, Logroño.

Ministerio de Educación, Cultura y Deporte-Instituto Nacional de Tecnologías Educativas y de Formación del Profesorado [Intef]. (2017). Resumen Informe Horizon. Edición 2017. Educación Superior. España: Ministerio de Educación, Cultura y Deporte-Instituto Nacional de Tecnologías Educativas y de Formación del Profesorado.

Mojerón, J. F. (2018). La realidad aumentada y la didáctica educativa. (Tesis de maestría). Universidad Técnica de Ambato, Ambato. Recuperado de http://repositorio.uta.edu.ec/jspui/handle/123456789/28916.

Pérez, Y. y Chamizo, J. (2016). Análisis curricular de la enseñanza química en México en los niveles preuniversitarios. Parte II: La educación media superior. Educación Química, 27(3), 182-194. Recuperado de https://doi.org/10.1016/j.eq.2015.12.001.

Prendes, E. (2015). Realidad aumentada y educación: análisis de experiencias prácticas. Píxel-Bit. Revista de Medios y Educación, (46), 187-203.

Sector de Educación de la Oficina de la Unesco en Montevideo. (2009). Experiencias de enseñanza y de aprendizaje para compartir. Montevideo, Uruguay: Sector de Educación de la Oficina de la Unesco en Montevideo. Recuperado de https://unesdoc.unesco.org/ark:/48223/pf0000182698.

Vásquez, R. (2017). Desarrollo de un plan de implementación de tecnología de Realidad Mixta en el mercado ecuatoriano. (Tesis de grado). Universidad Católica de Ecuador, Quito. http://repositorio.puce.edu.ec/handle/22000/14427.

Villa, A. y Villa, O. (2007). El aprendizaje basado en competencias y el desarrollo de la dimensión social en las universidades. Educar, (40), 15-48. Recuperado de https://www.raco.cat/index.php/Educar/article/view/119469. 
Villafañe, C. (2009). Diseño instruccional. Educación y tecnología. Colombia: Ministerio de Educación Nacional. Recuperado de https://es.slideshare.net/cvillafa1/diseoinstruccional-1750371.

Villarejo, A. B. (2019). Análisis motivacional respecto al aprendizaje a través de la realidad aumentada en la enseñanza de ciclos formativos. Revista Interuniversitaria de Investigación en Tecnología Educativa, (6). Recuperado de https://doi.org/10.6018/riite.380861 


\section{Anexo}

Tabla 4. Cuestionario sobre arquitectura de hardware

Instrucciones. Estimado estudiante, la intención del cuestionario es conocer los conocimientos previos sobre la materia de Arquitectura de Hardware, el cual no tiene ningún valor en la evaluación del semestre correspondiente.

1. ¿Qué es la informática?

a) Es la ciencia que estudia los componentes físicos de una computadora.

b) Es la ciencia aplicada que abarca el estudio y aplicación del tratamiento automático de la información.

c) Es la ciencia que estudia el uso de Word, Excel, Power Point, etc.

2. Las TIC involucran teléfonos, consolas de videojuegos, cámaras, laptops, tabletas, etcétera.

a) Verdadero

b) Falso

3. ¿Dispositivo que permite la salida de información?

a) Mouse

b) Teclado

c) Impresora

4. El procesador es:

a) El cerebro de una computadora

b) El chip central de la computadora

c) La capacidad de almacenamiento

d) El sistema operativo

5. Los periféricos se clasifican en:

a) De entrada, salida, de almacenamiento y mixtos

b) De entrada y de subida

c) Mixtos, de subida y almacenamiento

d) De subida y de bajada

6. Mencione cada una de las partes de la motherboard:

7. Existen dos tipos de memorias principales:

a) RAM y ROM

b) RAM y PROM

c) ROM y PROM 
d) BROM y Rambus

8. Los dispositivos de salida son todos aquellos mediante los cuales el computador:

a) Funciona correctamente

b) Maneja efectivamente los dispositivos de entrada

c) Permite ver toda la información en bruto

d) Entrega al exterior la información procesada

9. Es el componente más importante de la computadora, ya que es el cerebro que controla y administra información, y ejerce el control de la computadora.
a) $\mathrm{CPU}$
b) Monitor
c) Teclado

10. Menciona qué son los elementos de entrada a una computadora.

a) Son programas en espera al ser ejecutados

b) Son los que te permiten abrir Internet

c) Son los que permiten dar entrada al CPU

11. Las características de gran importancia de un microprocesador son:

a) Tecnología de fabricación.

b) Aceleración de gráficos.

c) Velocidad de procesamiento de datos.

12. ¿Alguna vez ha ensamblado una computadora?
a) Sí
b) No
c) Algunas veces

13. ¿Identifica los pasos y las medidas correctas para ensamblar una computadora?
a) Sí
b) No
c) Los recuerdo ligeramente 
Tabla 5. Modelo de TAM

Objetivo: Comprobar el grado de aceptación tecnológica de la realidad mixta (RM)como uso didáctico, para el aprendizaje práctico de los estudiantes.

Considere la siguiente escala: En desacuerdo (1), Desacuerdo (2), Indiferente (3), De acuerdo (4), Totalmente de acuerdo (5).

\begin{tabular}{|c|c|c|c|c|c|}
\hline Facilidad de uso percibida con RM & & & & & \\
\hline Escala & 1 & 2 & 3 & 4 & 5 \\
\hline 1. Fue fácil interactuar con componentes de RM. & & & & & \\
\hline 2. He encontrado dificultad en realizar tareas en el mundo virtual. & & & & & \\
\hline Usabilidad de realidades RM & & & & & \\
\hline Escala & 1 & 2 & 3 & 4 & 5 \\
\hline 1. Fue fácil entender el contenido con el uso de RM. & & & & & \\
\hline 2. Los componentes visuales de RM son interactivos. & & & & & \\
\hline 3. Los componentes visuales de RM son complejos. & & & & & \\
\hline Utilidad de aprendizaje y motivación con las RM & & & & & \\
\hline Escala & 1 & 2 & 3 & 4 & 5 \\
\hline $\begin{array}{l}\text { 1. Me resultó fácil comprender el contenido de Arquitectura de } \\
\text { hardware con las RM. }\end{array}$ & & & & & \\
\hline 2. Me gustaría realizar actividades con el uso de RM en clases. & & & & & \\
\hline $\begin{array}{l}\text { 3. Me resultó fácil conocer las partes de una computadora con } \\
\text { RM. }\end{array}$ & & & & & \\
\hline 4. Me resultó interesante usar las $\mathrm{RM}$ para mi aprendiz & & & & & \\
\hline
\end{tabular}


Tabla 6. Lista de cotejo

\begin{tabular}{|l|l|l|l|}
\hline Procedimiento de ensamblaje & Sí & No & Observaciones \\
\hline $\begin{array}{l}\text { Identifica el proceso de seguridad para el } \\
\text { ensamblaje correcto. }\end{array}$ & & & \\
\hline $\begin{array}{l}\text { Identifica cada uno de los componentes: Disco } \\
\text { duro, tarjeta RAM, tarjeta gráfica, etcétera. }\end{array}$ & & & \\
\hline Inicia procedimiento. & & & \\
\hline $\begin{array}{l}\text { Coloca cada una de las partes en el lugar } \\
\text { correspondiente: }\end{array}$ & & & \\
\hline Realiza pruebas de funcionalidad. & & & \\
\hline Ensambla de manera satisfactoria. & & & \\
\hline
\end{tabular}

